\title{
Dermatophytes Species Isolated of HIV-Infected Patients Identified by ITS-RFLP and ITS Region Sequencing from Triângulo Mineiro, Minas Gerais State of Brazil
}

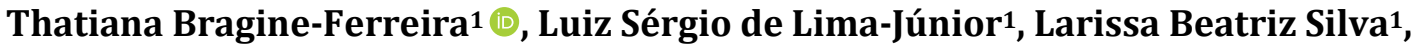 \\ Leonardo Eurípedes Andrade-Silva1, Delio José Mora1, Bruno Siqueira Prudente1, \\ Kennio Ferreira-Paim², Fabiana Almeida Araújo Santos ${ }^{3}$, Luiz Ricardo Goulart ${ }^{3}$, \\ Mario León Silva-Vergara ${ }^{*}$ (i)
}

\begin{abstract}
${ }^{1}$ Infectious Diseases Unit, Internal Medicine Department, Triângulo Mineiro Federal University, Uberaba, Brazil ${ }^{2}$ Department of Microbiology, Triângulo Mineiro Federal University, Uberaba, Brazil

${ }^{3}$ Laboratory of Nanobiotechnology, Institute of Biotechnology, Federal University of Uberlândia, Uberlândia, Brazil

Email: *marioleon.dip@mednet.com.br
\end{abstract}

How to cite this paper: Bragine-Ferreira, T., de Lima-Júnior, L.S., Silva, L.B., Andrade-Silva, L.E., Mora, D.J., Prudente, B.S., Ferreira-Paim, K., Santos, F.A.A., Goulart, L.R. and Silva-Vergara, M.L. (2019) Dermatophytes Species Isolated of HIV-Infected Patients Identified by ITS-RFLP and ITS Region Sequencing from Triângulo Mineiro, Minas Gerais State of Brazil. $A d$ vances in Microbiology, 9, 790-803. https://doi.org/10.4236/aim.2019.99048

\section{Received: July 25, 2019}

Accepted: September 2, 2019

Published: September 5, 2019

Copyright $\odot 2019$ by author(s) and Scientific Research Publishing Inc. This work is licensed under the Creative Commons Attribution International License (CC BY 4.0).

http://creativecommons.org/licenses/by/4.0/

(c) (i) Open Access

\begin{abstract}
Dermatomycoses are a group of diseases worldwide distributed and affect skin, hair and nails. Several fungal species can be envolved and keratinophilic fungi belonging to the genera Trichophyton, Microsporum and Epidermophyton are very common. These infections are globally distributed and their incidence has progressively increased. Despite their high incidence, few studies have been carried out in HIV-infected patients, regarding frequency, clinical presentations, and species identification. This study aimed to evaluate some epidemiological and clinical aspects of dermatophytosis in HIV-infected patients and to attempt phenotypical and molecular characteristics of their agents. Of 398 patients included, 306 were HIV-infected. Clinical data and samples of skin, hair and nail lesions were simultaneously obtained. The dermatophytes yielded in culture were phenotypically identified and evaluated by ITS-RFLP and ITS (Internal Transcribed Space) sequencing. Dermatophytes frequency in HIV-infected patients with cutaneous lesions was $11.76 \%$ similar to the $15.22 \%$ observed in non-HIV individuals. Trichophyton rubrum was the most common isolated species in both groups. Throught ITS-RFLP, 29/32 (90.63\%) of dermatophytes isolates were identified at the species level. From the 10 isolates randomly selected for ITS sequencing, seven confirmed the ITS-RFLP results while three Trichophyton sp. were not identified as dermatophytes. The identification of these species in HIV-infected patients is highly desirable in order to improve the knowledge of their fre-
\end{abstract}


quency, geographical distribution and relation to clinical presentation.

\section{Keywords}

Dermatophytosis, Dermatophytes, ITS-RFLP, ITS-Sequencing, HIV-Infected Patients, Trichophyton rubrum

\section{Introduction}

Dermatomycoses are common cutaneous infections of keratinized tissues, caused by several fungal species which invade the corneous layer of skin, hair and nails. Among these, keratinophilic fungi belonging to the genera Trichophyton, Microsporum and Epidermophyton are very common. These infections are globally distributed and their incidence has progressively increased [1]. According to the World Health Organization (WHO), approximately 20\% - 25\% of the world population has some kind of cutaneous fungal infection [1] [2] [3].

These infections can have acute or chronic clinical presentation, depending on the degree of inflammation and it is hypothesized that these mycoses trend to be more frequent and severe in immunocompromised individuals [4] [5] [6]. The HIV infection is one of the most important risk factors to the occurrence of fungal infections and due to the scarcity of data, the prevalence, clinical features, diagnosis and outcome of the dermatomycosis in HIV-infected individuals are still unclear [7]. Moreover, cellular immunity is pivotal in the control of these infections and Th1 and Th2 responses balance can determinate the progression of the infection in such patients [8] [9].

The taxonomy of dermatophytes remained confused for a long time, until the discovery of the sexual reproduction in keratinophilic species, which led to the introduction of the biological concept of species [10]. With the advent of molecular biology, conventional morphological taxonomy was influenced by data obtained DNA sequencing which improved the taxonomic system [11]. One of the sequencing targets is the Internal Transcribed Spacer (ITS) region of ribosomal DNA, which is considered the main barcode of the fungal kingdom [12] [13]. Furthermore, the sequencing of different genes, specially using the genealogical concordance phylogenetic species recognition (GCPSR) can be useful to improve fungal diagnosis, biodiversity range, species conservation and taxonomy accuracy [11].

The present report aimed to evaluate some epidemiological and clinical aspects of dermatophytosis in HIV-infected patients and to attempt characterizes phenotipic and molecular aspects of their etiological agents in a teaching hospital from Brazil.

\section{Population and Methods}

\subsection{Population}

This study was carried out at the Infectious Diseases Outpatient Unit in a Bra- 
zilian Teaching Hospital from August 2014 to December 2015. A total of 398 adult patients of both genders were randomicaly recruited and divided into three groups: I composed by $153 \mathrm{HIV}$-infected individuals with skin, hair or nail lesions, II composed by $153 \mathrm{HIV}$-infected without apparent clinical lesions in these sites and III composed by 92 non-HIV-patients with clinical lesions similar to group I seen at the Dermatology Outpatient Clinic of the same hospital. The inclusion criteria were: both genders, age over 18 years, that was receiving antiretroviral therapy and agree to participate of the study. Those who referred antifungal use within 30 days prior to the recruitment or disagreed to participate were excluded.

Epidemiological and clinical data of the HIV-infected patients with dermatomycosis were registered and a clinical exam was performed. Nail and cutaneous samples were obtained by scraping while hair fragments were obtainied by removing a small amount of hair containing their bulb. Concomitantly, nail scrapings samples of the $153 \mathrm{HIV}$-infected without clinical lesions were obtained.

These clinical samples were examined by direct microscopic using potassium hydroxide $20 \%(\mathrm{KOH})$ in order to identify fungal structures. Then, they were seeded in Sabouraud Dextrose Agar added with $0.05 \mathrm{mg} \cdot \mathrm{mL}^{-1}$ chloramphenicol and Mycosel Agar, incubated at $28^{\circ} \mathrm{C} \pm 2{ }^{\circ} \mathrm{C}$ and observed for up to 30 days. Identification of the yielded filamentous fungi was carried out using the microculture technique in Potato Dextrose Agar for seven days at room temperature.

\subsection{Molecular Identification of Isolates}

The DNA extraction was performed by the phenol-chloroform method [14]. Then, a PCR reaction of the ITS region of ribosomal DNA was performed using ITS-1 and ITS-4 primers as previously described with modifications [15]. Briefly, each reaction contained a final volume of $50 \mu \mathrm{L}$ with $50 \mathrm{ng}$ of template DNA, 5 $\mu \mathrm{L}$ of the 1X PCR buffer (10 mM Tris- $\mathrm{HCl} \mathrm{pH} 8.3,50 \mathrm{mM} \mathrm{KCl}, 0.1 \%, 1.5 \mathrm{mM}$ $\mathrm{MgCl}_{2}$ ), 0.25 mM dNTP's (dATP, dCTP, dGTPe dTTP), 1.25 U Taq DNA polymerase (Invitrogen, Brazil) and 70 pmol of each primer. The PCR was performed under the following conditions: initial denaturation at $94^{\circ} \mathrm{C}(3 \mathrm{~min})$ followed by 29 cycles with denaturation at $94^{\circ} \mathrm{C}(30 \mathrm{~s})$, association at $57^{\circ} \mathrm{C}(30 \mathrm{~s})$, extension at $72^{\circ} \mathrm{C}(45 \mathrm{~s})$ and one cycle 10 minutes at $72^{\circ} \mathrm{C}$.

Next, the DNA was digested using the restriction enzyme MvaI Fast Digest (Thermo Scientific, USA) according to the manufacturer's instructions. The fragments were detected by electrophoresis in $2 \%$ agarose gel at $80 \mathrm{~V}$ for 2 hours. The profiles were visually identified by comparison with the restriction patterns already described [16] [17]. The strain ATCC MYA4439 of Trichophyton interdigitale was used as a positive control.

The amplified DNA of ten randomly selected isolates was submitted to the sequencing reaction using the BigDye terminator 3.1 reagent kit (Applied Biosystems, Foster City, CA, USA). The sequences obtained were analyzed in the software Chromas-pro 1.5 and then compared with the available data in the $\mathrm{Na}$ tional Center of Biotechnological Information (NCBI-Nacional Center for Biotechnolgy Information), including sequences of reference strains. Multiple align- 
ments were performed in the Clustal W software [18]. The best model used in the phylogenetic tree was chosen from the software JModel test using the Bayesian Information Criterion (BIC). The Tamura Nei substitution model with Gamma distribution was the best model for the aligned dataset and used to construct the phylogenetic tree. The phylogenetic analysis was performed in the MEGA 7.0 software with the Maximum likelihood method [19]. The Bootstrap analysis with 1000 replicates was used to support the tree nodes.

\subsection{Statistical Analysis}

Data analysis was performed using Epi Info software (Statistical Package for the Social Sciences, version 7.0, USA) and GraphPad Prism (version 5.0, USA). The verification of normal distribution of the quantitative variables was done by the Shapiro-Wilk test. Continuous variables with normal distribution were expressed as mean \pm standard deviation and those with ab-normal distribution were expressed in median, with maximum, minimum and percentiles values. Mann-Whitney and Fisher exact tests were used to evaluate the differences and frequencies among groups. Values of $p<0.05$ were considered statistically significant.

The project was approved by the Research Ethics Board of the Triângulo Mineiro Federal University in Uberaba, Minas Gerais State, Brazil under protocol number: 1716 .

\section{Results}

Of $306 \mathrm{HIV}$-infected patients, 178 (58.17\%) were male (mean age: $43.86 \pm 12.24$ years) (Table 1). Of $153 \mathrm{HIV}$ patients with cutaneous, hair or nails lesions, in 61 (39.87\%) at least one fungal structure was observed by direct examination whereas in $72(47.06 \%)$ of cases a positive fungal culture was obtained. The presence of skin, hair or nail lesions was significantly associated with the chance to obtain a positive fungal direct exam or culture $(\mathrm{p}<0.001)$ (Table 2$)$.

Of 92 non-HIV patients evaluated, 55 (59.78\%) were female (mean age: 52.64 \pm 13.63 years). Of these, $43(46.74 \%)$ and $48(52.17 \%)$ presented a fungal positive direct exam and culture, respectively (Table 2).

Among the recovered fungal isolates, 32 were phenotypically identified as dermatophytes, of which 18 (56.25\%) were obtained from HIV-infected patients while the remaining 14 (43.75\%) were from non-HIV patients (Table S1).

The frequency of dermatophytes in HIV-infected patients with clinical lesions (11.76\%) was similar to that observed in non-HIV patients $(15.22 \%)(\mathrm{p}=$ 0.4406). Trichophyton rubrum was predominant followed by Trichophyton interdigitale in both groups (Table S1).

Of the 18 HIV-infected patients with dermatophytes isolation, 14 (77.78\%) were males (mean age: 48.22 years) while the $9(64.29 \%)$ of the 14 non-HIV patients with isolated dermathophytes were female (mean age: 50.93 years). Most individuals were from urban areas and had a low scholar level. Other epidemiological and clinical data are described in Table 3 and Table S1. 
Table 1. Main demographic, epidemiological and clinical data of the HIV-infected and Non-HIV patient groups.

\begin{tabular}{|c|c|c|c|}
\hline & & $\begin{array}{c}\text { HIV-infected } \\
\text { patients }(n=306)\end{array}$ & $\begin{array}{l}\text { Non-HIV patients } \\
(\mathrm{n}=92)\end{array}$ \\
\hline Age (years) & Average $\left( \pm \mathrm{SD}^{* \star}\right)$ & $43.86( \pm 12.24)$ & $52.64( \pm 13.63)$ \\
\hline Gender & Male & $178(58.17 \%)$ & $37(40.22 \%)$ \\
\hline \multirow[t]{7}{*}{ Educational level } & Complete elementary school & $46(15.06 \%)$ & $4(4.35 \%)$ \\
\hline & Incomplete elementary school & $168(54.90 \%)$ & $32(34.78 \%)$ \\
\hline & Complete high school & $44(14.38 \%)$ & $32(34.78 \%)$ \\
\hline & Incomplete high school & $20(6.54 \%)$ & $4(4.35 \%)$ \\
\hline & Complete college & $8(2.61 \%)$ & $9(9.78 \%)$ \\
\hline & Incomplete college & $6(1.96 \%)$ & $5(5.43 \%)$ \\
\hline & None & $14(4.58 \%)$ & $6(6.52 \%)$ \\
\hline \multirow[t]{6}{*}{ Profession } & Retired & $68(22.22 \%)$ & $20(21.74 \%)$ \\
\hline & Housewife & $53(17.32 \%)$ & $21(22.83 \%)$ \\
\hline & Farmer & $31(10.31 \%)$ & $3(3.26 \%)$ \\
\hline & Student & $5(1.63 \%)$ & $1(1.09 \%)$ \\
\hline & Others & $111(36.27 \%)$ & $45(48.91 \%)$ \\
\hline & None & $38(12.42 \%)$ & $2(2.17 \%)$ \\
\hline \multirow[t]{2}{*}{ Domicile } & Urban & $275(89.87 \%)$ & $85(92.39 \%)$ \\
\hline & Rural & $31(10.13 \%)$ & $7(7.61 \%)$ \\
\hline Swimming/pool practices* & - & $42(13.86 \%)$ & $22(23.91 \%)$ \\
\hline Skin/nail lesions & - & $153(50 \%)$ & $92(100 \%)$ \\
\hline \multirow[t]{3}{*}{$\begin{array}{l}\text { Previous history of } \\
\text { skin/nail lesions* }\end{array}$} & Yes & $134(44.23 \%)$ & $62(67.39 \%)$ \\
\hline & No & $159(52.47 \%)$ & $28(30.43 \%)$ \\
\hline & Unknown & $10(3.3 \%)$ & $2(2.17 \%)$ \\
\hline Underlying diseases & - & $130(42.48 \%)$ & $50(54.35 \%)$ \\
\hline \multirow[t]{4}{*}{$\begin{array}{l}\text { Frequency of the contact } \\
\text { with animals* }\end{array}$} & Common & $157(51.82 \%)$ & $35(38.04 \%)$ \\
\hline & Often & $26(8.58 \%)$ & $10(10.87 \%)$ \\
\hline & Occasional & $13(4.29 \%)$ & $8(8.70 \%)$ \\
\hline & None & $107(35.31 \%)$ & $39(42.39 \%)$ \\
\hline
\end{tabular}

${ }^{\star}$ Three patients from the HIV-infected group were excluded due to unavailable data; ${ }^{* * S D}$ : standard deviation.

Table 2. Mycological diagnosis according with the presence or absence of skin, hair or nail lesions in HIV-infected patients.

\begin{tabular}{cccc}
\hline & $\begin{array}{c}\text { Presence of lesions } \\
\mathbf{n}(\%)\end{array}$ & $\begin{array}{c}\text { Absence of lesions } \\
\mathbf{n}(\%)\end{array}$ & $\mathbf{p}$ \\
\hline KOH+ & $61(39.87)$ & $12(7.84)$ & $<0.0001$ \\
KOH- & $92(60.13)$ & $141(92.16)$ & - \\
Culture+ & $72(47.06)$ & $31(20.26)$ & $<0.0001$ \\
Culture- & $81(52.94)$ & $122(79.74)$ & - \\
Total & $153(100)$ & $153(100)$ & - \\
\hline
\end{tabular}

${ }^{*} \mathrm{KOH}$ : potassium hydroxide $20 \%$. 
Table 3. Epidemiological and clinical data of patients with positive culture for dermatophytes.

\begin{tabular}{cccc}
\hline & & $\begin{array}{c}\text { HIV-infected with } \\
\text { cutaneous lesions } \\
\mathbf{n}=18(\%)\end{array}$ & $\begin{array}{c}\text { Non-HIV patient } \\
\mathbf{n}=\mathbf{1 4}(\%)\end{array}$ \\
\hline Age (years) & Average (range) & $48.22(32-63)$ & $50.93(37-71)$ \\
Gender & Male & $14(77.78)$ & $5(35.71)$ \\
History of contact with animals & - & $10(55.56)$ & $7(50)$ \\
Underlying diseases & - & $7(38.89)$ & $9(64.29)$ \\
Previous skin/nail lesions & - & $13(72.22)$ & $10(71.43)$ \\
Previous antifungal therapy & Yes & $8(44.44)$ & $5(35.71)$ \\
& No & $8(44.44)$ & $8(57.14)$ \\
T CD4 ${ }^{+}$cells/mm ${ }^{3}$ & Not reported & $2(11.11)$ & $1(7.14)$ \\
& $<200$ & $8(44.44)$ & NA \\
& 200 to 499 & $2(11.11)$ & NA \\
& $\geq 500$ & $8(44.44)$ & NA \\
& $<50$ & $8(44.44)$ & NA \\
Viral load (copies of RNA/mL) & 50 to 100,000 & $7(38.89)$ & NA \\
& $>100.000$ & $3(16.67)$ & NA \\
\hline
\end{tabular}

NA: not available.

\subsection{ITS-RFLP}

Of 32 isolated dermatophytes, 29 (90.63\%) were identified at the species level by the ITS-RFLP profiles. All 18 isolates phenotypically characterized as T. rubrum were confirmed by ITS-RFLP. Of the six isolates previously identified as $T$. interdigitale, four presented the typical profile of this species while two were reclassified as T. rubrum (Figure 1). Two isolates previously identified as Microsporum canis and T. verrucosum were confirmed as well (data not shown).

From the six isolates (D04, D05, D07, D22, D30 and D32) previously identified as Trichophyton sp., three (D04, D30 and D32) were confirmed as T. rubrum by the ITS-RFLP (Figure 1). The remaining isolates did not present any pattern of digestion by this technique.

\subsection{Sequencing of the ITS Region and Phylogenetic Analysis}

Ten $(31.25 \%)$ of isolates were randomly selected for ITS sequencing. The generated ITS fragments presented approximately $630 \mathrm{bp}$. Of these, seven (70\%) were adequately confirmed being five $T$. rubrum and two $T$. interdigitale. The sequences presented $97 \%$ to $100 \%$ of identity in relation to clinical isolates from USA, Australia, India, China, Russia and Japan.

The comparison of these sequences with the 29 reference sequences from GenBank, demonstrated that the T. rubrum isolates herein identified appear to have a greater phylogenetic homegeneity in comparison to the CBS 392.58 reference 


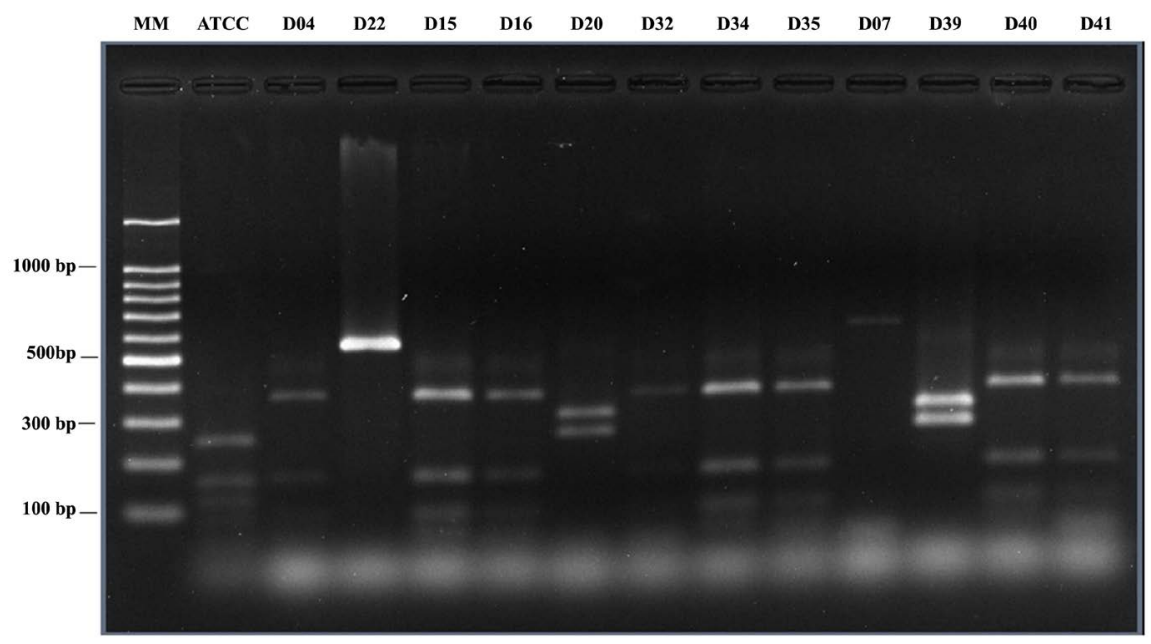

Figure 1. Agarose gel (2\%) representative of the band pattern after digestion with Mva I. ATCC: MYA4439 Trichophyton interdigitale; D04, D15, D16, D32, D34, D35, D40, D41: Trichophyton rubrum; D22, D07: not digest samples; D20, D39: excluded isolates. MM: LowRanger 100 bp DNA Ladder (NorGen Biotek, Canada). Color: Ethidium Bromide $(0.5 \mu \mathrm{g} / \mathrm{mL})$.

strain. Otherwise, the isolates D33 and D38 were grouped together with the anthropophilic strains of T. mentagrophytes complex (Figure 2).

The remaining three isolates formerly identified as Trichophyton sp. by their phenotypic aspects were identified within Ascomycetes. Lecanicillium aphanocladii (MH346472), Preussia sp. (MH346502) and Montagnulaceae sp. (MH348207).

\section{Discussion}

Dermatophytes comprise a group of pathogenic filamentous fungi that cause cutaneous mycoses and due to their ubiquity in nature and high transmissibility, the infections caused by these agents are often considered a neglected problem [20] [21]. Currently, HIV infection is considered one of the major global public health problems and a wide gamut of diseases with cutaneous involvement in HIV-infected patients has been already reported. Due to the immunosuppresion, atypical skin lesions can lead to clinical misdiagnosis and treatment difficulties [22] [23].

The relationship between dermatophytosis and HIV infection remains poorly understood, regarding their frequency, causative species and therapeutical outcome [9] [24]. Of $306 \mathrm{HIV}$-infected patients herein evaluated, 50\% presented cutaneous, hair or nail lesions, often with atypical and heterogeneous appearance. Despite fungal ethiology had been suspected, other causes should be excluded and for this direct exam, culture and molecular methods are pivotal to improve the diagnosis accuracy and therapeutical decision [25].

In the present report, $39.87 \%$ and $47.06 \%$ of HIV-infected patients with skin, hair or nail lesions presented direct exam and positive fungal culture respectively. Whereas in non-HIV individuals, $46.74 \%$ and $52.17 \%$ of cases presented direct exam and culture positive respectively. These figures were similar with those 


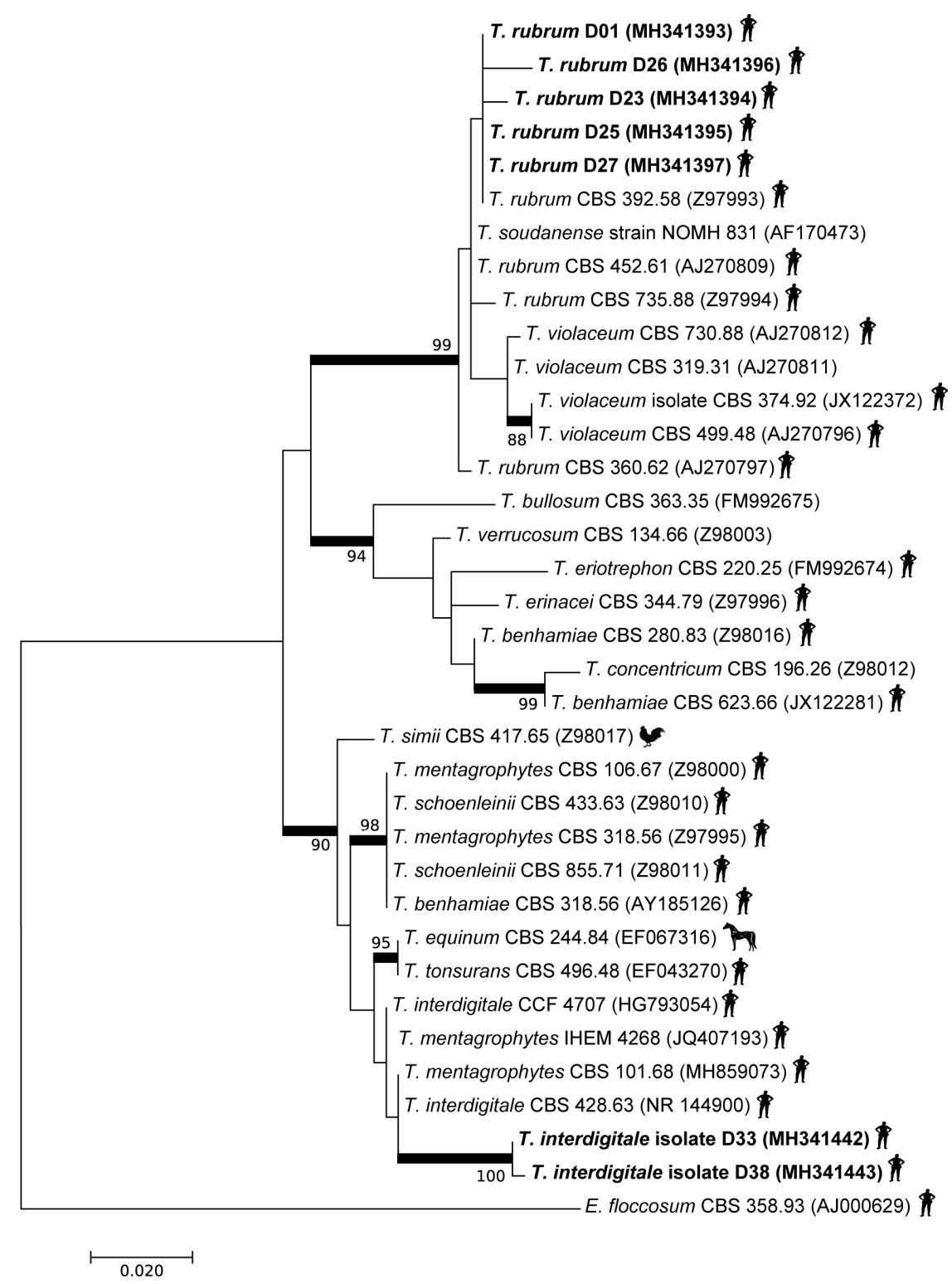

Figure 2. The evolutionary history of the phylogenetic tree was inferred by using the Maximum Likelihood method based on the Tamura-Nei model. The tree with the highest log likelihood (-1415.8245) is shown. A discrete Gamma distribution was used to model evolutionary rate differences among sites $(5$ categories $(+G$, parameter $=0.3745)$ ). The tree is drawn to scale, with branch lengths measured in the number of substitutions per site. All positions containing gaps and missing data were eliminated. There were a total of 437 positions in the final dataset and 36 isolates were tested. The isolates are described according species of Trichophyton, strain ID, GenBank number, and the host from where they were recovered (e.g. human, horse, or chicken). The isolates described in bold are described in this study. The reference strain Epidermophyton floccosum CBS 358.93 was used as outgroup. The bootstrap values above $80 \%$ are described next to the branches.

reported from India, where the positivity rate of direct microscopy and culture were respectively $49 \%$ and $51 \%$ in 100 non-HIV patients with clinical suspicious of dermatophytosis [26].

The frequency of dermatophytes isolation between HIV-infected patients and 
non-HIV individuals was similar, a finding in line with another report where 105 HIV-infected patients and 63 non-HIV individuals were evaluated. According to the authors, there was no correlation between the $\mathrm{T} \mathrm{CD} 4^{+}$cell count and the prevalence of dermatophytosis in HIV patients [7].

Although the relation of dermatophytosis and HIV-infected patients is still unclear, some authors pointed out an inverse relationship between $\mathrm{T} \mathrm{CD} 4^{+}$cells count and the frequency and extension of cutaneous lesions in these individuals [9]. Most patients herein evaluated exhibited high $\mathrm{T} \mathrm{CD} 4^{+}$cell counts and undetectable viral load values, which reinforce that they presented adequate adhesion to antiretroviral therapy and partially could explain the low frequency of dermatophytes recovered despite 306 patients were evaluated. Herein and as described for other mycosis, the HIV infection can change the clinical presentation without alters the rate incidence. Maybe, the stratification of $\mathrm{CD} 4^{+}$values and the inclusion of patients with advanced immunodeficiency could identify different risk categories for dermatophytes infection.

Regarding the anatomic site, the toenails and feet were the sites where dermatophytes were more frequently isolated. The severity of the nail involvement with chronic and distrophic changes and a poor antifungal response is a common clinical observation in these patients. This finding agrees with literature data and confirms the results from a recent Brazilian survey where onicomycosis and Tinea pedis were the most common clinical pictures in $84 \mathrm{HIV}$-infected patients evaluated [27]. In contrast, another study from the same country described a high frequency (70\%) of Tinea corporis in 20 HIV-infected patients [28].

Most dermatophytes species herein identified belong to the Trichophyton genus which is in line with other reports that point out $90 \%$ of the dermatophytosis around the world are caused by species of this genus [29] [30]. Although $T$. rubrum is found as the main agent of dermatophytosis in HIV-infected patients, it presents global distribution and also is the most common agent recovered from clinical specimens [7] [27].

The $T$. verrucosum isolate was confirmed by ITS-RFLP and recovered from a patient who referred frequent contact with domestic animals. Despite this zoophilic species is an uncommon agent in humans, its isolation could be clinically relevant since it was recovered from an HIV-infected individual. This species has been commonly associated with farm activities and cattle are known as the main infection source. However, accidental transmission can also occur by contaminated objects [31] [32].

The therapy to superficial mycoses in HIV/AIDS patients is usually started with topical antifungals, whereas the use of systemic drugs is recommended in cases where there is no improvement or when lesions are extensive, deep or widespread. Several reports have pointed out that oral medicaments such as terbinafine, intraconazole or fluconazole are the most appropriate therapeutic options for these individuals. According with other authors, they must be prescribed from two to six weeks except those who present oniocomycosis and re- 
quire more time of treatment [9] [24]. Patients with dermatomycosis herein described received topical or oral itraconazole with clinical remission of their lesions except those who presented nail involvement and exhibited a poor response.

Despite, culture continues to be one of the most used methods for mycological diagnosis, the ITS-RFLP allowed the identification of $90.63 \%$ of the isolated dermatophytes at the species level. The obtained band patterns were variable in size among the different species, which allowed the visual differentiation among T. rubrum, T. interdigitale, $M$. canis and $T$. verrucosum [17]. The T. rubrum complex includes species with different phenotypic characteristics, however this variability has not been homogeneously observed in molecular studies. In these cases, the restriction pattern from other DNA regions or the use of additional enzymes could increase the accuracy of the method [16] [33]. Recently, a wholegenome analysis showed a clonal population profile of this species, which may explain the genetic homogeneity of the complex [34].

In accordance to the current taxonomy of dermatophytes, the anthropophilic isolates are separated from the zoophilic ones constituting the $T$. mentagrophytes species complex [11]. The $T$. interdigitale isolates herein identified presented the same restriction pattern on ITS-RFLP and were grouped together with the anthropophilic ones in the phylogenetic analysis. A recent report with 60 clinical isolates of dermatophytes showed that the most of them belonged to the anthropophilic variant of $T$. mentagrophytes complex [35].

Despite, the DNA sequencing is a reliable technique for fungal identification species and the ITS region is relatively conserved in dermatophytes species, the combination of different genes sequencing of these species by MLST (Multilocus Sequence Typing) undoubtedly is pivotal to improve their identification [11].

Despite the low number of isolates evaluated, the results herein showed that identification based on morphological characteristics of dermatophytes species presents high level of complexity and accuracy limitations, although it is still one of the most used method in the context of clinical mycological diagnosis. Otherwise the ITS-RFLP appears to be useful and reliable tool to identify and to differentiate dermatophytes at the species level. In addition, this technique is still cheaper and simpler than any sequencing method, especially when taking into account limited-resource settings.

Moreover, the sequencing is considered the ideal method to identify the dermatophytes with more precision at the species level and to observe their intraspecific variability. However, logistical and economical issues around the world remain as the main obstacle for its wide implementation as well.

\section{Funding}

This work was supported by Fundação de Amparo a Pesquisa de Minas Gerais (FAPEMIG)-grant \#APQ-02633-16. The funders had no role in study design, data collection and analysis, decision to publish, or preparation of the manu- 
script.

\section{Ethical Approval}

All procedures performed in studies involving human participants were in accordance with the ethical standards of the institutional and/or national research committee and with the 1964 Helsinki declaration and its later amendments or comparable ethical standards. Informed consent was obtained from all individual participants included in the study.

\section{Authors' Contributions}

T.B.F. and M.L.S.V. conceived the study; T.B.F., L.S.L.J. and B.S.P. collected the data; T.B.F. and L.B.S. performed experiments; T.B.F., L.B.S., L.E.A.S., K.F.P. and M.L.S.V analyzed data; F.A.A.S. and L.R.G. contributed new methods; T.B.F., D.J.M., K.F.P and M.L.S.V. led the writing.

\section{Conflicts of Interest}

There are no conflicts of interest to report.

\section{References}

[1] Ameen, M. (2010) Epidemiology of Superficial Fungal Infections. Clinics in Dermatology, 28, 197-201. https://doi.org/10.1016/j.clindermatol.2009.12.005

[2] Hube, B., Hay, R., Brasch, J., Veraldi, S. and Schaller, M. (2015) Dermatomycoses and Inflammation: The Adaptive Balance between Growth, Damage, and Survival. Journal de Mycologie Médicale, 25, e44-e58.

[3] Silva, L.B., de Oliveira, D.B., da Silva, B.V., de Souza, R.A., da Silva, P.R., FerreiraPaim, K., et al. (2014) Identification and Antifungal Susceptibility of Fungi Isolated from Dermatomycoses. Journal of the European Academy of Dermatology and Venereology, 28, 633-640. https://doi.org/10.1111/jdv.12151

[4] Criado, P.R., Oliveira, C.B., Dantas, K.C., Takiguti, F.A., Benini, L.V. and Vasconcellos, C. (2011) Superficial Mycosis and the Immune Response Elements. Anais Brasileiros de Dermatologia, 86, 726-731. https://doi.org/10.1590/S0365-05962011000400015

[5] Marconi, V.C., Kradin, R., Marty, F.M., Hospenthal, D.R. and Kotton, C.N. (2010) Disseminated Dermatophytosis in a Patient with Hereditary Hemochromatosis and Hepatic Cirrhosis: Case Report and Review of the Literature. Medical Mycology, 48, 518-527. https://doi.org/10.3109/13693780903213512

[6] Rouzaud, C., Hay, R., Chosidow, O., Dupin, N., Puel, A., Lortholary, O., et al. (2015) Severe Dermatophytosis and Acquired or Innate Immunodeficiency: A Review. Journal of Fungi, 2, 4. https://doi.org/10.3390/jof2010004

[7] Rodwell, G.E., Bayles, C.L., Towersey, L. and Aly, R. (2008) The Prevalence of Dermatophyte Infection in Patients Infected with Human Immunodeficiency Virus. International Journal of Dermatology, 47, 339-343. https://doi.org/10.1111/j.1365-4632.2008.03416.x

[8] Almeida, S.R. (2008) Immunology of Dermatophytosis. Mycopathologia, 166, $277-$ 283. https://doi.org/10.1007/s11046-008-9103-6

[9] Ramos, E.S.M., Lima, C.M., Schechtman, R.C., Trope, B.M. and Carneiro, S. (2010) 
Superficial Mycoses in Immunodepressed Patients (AIDS). Clinics in Dermatology, 28, 217-225. https://doi.org/10.1016/j.clindermatol.2009.12.008

[10] Kawasaki, M. (2011) Verification of a Taxonomy of Dermatophytes Based on Mating Results and Phylogenetic Analyses. Medical Mycology Journal, 52, 291-295.

https://doi.org/10.3314/mmj.52.291

[11] de Hoog, G.S., Dukik, K., Monod, M., Packeu, A., Stubbe, D., Hendrickx, M., et al. (2017) Toward a Novel Multilocus Phylogenetic Taxonomy for the Dermatophytes. Mycopathologia, 182, 5-31. https://doi.org/10.1007/s11046-016-0073-9

[12] Irinyi, L., Serena, C., Garcia-Hermoso, D., Arabatzis, M., Desnos-Ollivier, M., Vu, D., et al. (2015) International Society of Human and Animal Mycology (ISHAM)ITS Reference DNA Barcoding Database-The Quality Controlled Standard Tool for Routine Identification of Human and Animal Pathogenic Fungi. Medical Mycology, 53, 313-337. https://doi.org/10.1093/mmy/myv008

[13] Normand, A.C., Packeu, A., Cassagne, C., Hendrickx, M., Ranque, S. and Piarroux, R. (2018) Nucleotide Sequence Database Comparison for Routine Dermatophyte Identification by Internal Transcribed Spacer 2 Genetic Region DNA Barcoding. Journal of Clinical Microbiology, 56, 1-13. https://doi.org/10.1128/JCM.00046-18

[14] Makimura, K., Tamura, Y., Mochizuki, T., Hasegawa, A., Tajiri, Y., Hanazawa, R., et al. (1999) Phylogenetic Classification and Species Identification of Dermatophyte Strains Based on DNA Sequences of Nuclear Ribosomal Internal Transcribed Spacer 1 Regions. Journal of Clinical Microbiology, 37, 920-904.

[15] Ansari, S., Hedayati, M.T., Zomorodian, K., Pakshir, K., Badali, H., Rafiei, A., et al. (2016) Molecular Characterization and in Vitro Antifungal Susceptibility of 316 Clinical Isolates of Dermatophytes in Iran. Mycopathologia, 181, 89-95. https://doi.org/10.1007/s11046-015-9941-y

[16] Elavarashi, E., Kindo, A.J. and Kalyani, J. (2013) Optimization of PCR-RFLP Directly from the Skin and Nails in Cases of Dermatophytosis, Targeting the ITS and the 18S Ribosomal DNA Regions. Journal of Clinical and Diagnostic Research, 7, 646-651. https://doi.org/10.7860/JCDR/2013/5363.2873

[17] Rezaei-Matehkolaei, A., Makimura, K., Shidfar, M., Zaini, F., Eshraghian, M., Jalalizand, N., et al. (2012) Use of Single-Enzyme PCR-Restriction Digestion Barcode Targeting the Internal Transcribed Spacers (ITS rDNA) to Identify Dermatophyte Species. Iranian Journal of Public Health, 41, 82-94.

[18] Thompson, J.D., Higgins, D.G. and Gibson, T.J. (1994) CLUSTAL W: Improving the Sensitivity of Progressive Multiple Sequence Alignment through Sequence Weighting, Position-Specific Gap Penalties and Weight Matrix Choice. Nucleic Acids Research, 22, 4673-4680. https://doi.org/10.1093/nar/22.22.4673

[19] Kumar, S., Stecher, G. and Tamura, K. (2016) MEGA7: Molecular Evolutionary Genetics Analysis Version 7.0 for Bigger Datasets. Molecular Biology and Evolution, 33, 1870-1874. https://doi.org/10.1093/molbev/msw054

[20] Martinez-Rossi, N.M., Peres, N.T. and Rossi, A. (2017) Pathogenesis of Dermatophytosis: Sensing the Host Tissue. Mycopathologia, 182, 215-227. https://doi.org/10.1007/s11046-016-0057-9

[21] Zhan, P. and Liu, W. (2017) The Changing Face of Dermatophytic Infections Worldwide. Mycopathologia, 182, 77-86. https://doi.org/10.1007/s11046-016-0082-8

[22] Goodman, D.S., Teplitz, E.D., Wishner, A., Klein, R.S., Burk, P.G. and Hershenbaum, E. (1987) Prevalence of Cutaneous Disease in Patients with Acquired Immunodeficiency Syndrome (AIDS) or AIDS-Related Complex. Journal of the American Academy of Dermatology, 17, 210-220. 
https://doi.org/10.1016/S0190-9622(87)70193-5

[23] Huang, X.J., Li, H.Y., Chen, D.X., Wang, X.C., Li, Z.C., Wu, Y.S., et al. (2011) Clinical Analysis of Skin Lesions in 796 Chinese HIV-Positive Patients. Acta Dermato-Venereologica, 91, 552-556. https://doi.org/10.2340/00015555-1107

[24] Johnson, R.A. (2000) Dermatophyte Infections in Human Immune Deficiency Virus (HIV) Disease. Journal of the American Academy of Dermatology, 43, S135S142. https://doi.org/10.1067/mjd.2000.110631

[25] Khadka, S., Sherchand, J.B., Pokharel, D.B., Pokhrel, B.M., Mishra, S.K., Dhital, S., et al. (2016) Clinicomycological Characterization of Superficial Mycoses from a Tertiary Care Hospital in Nepal. Dermatology Research and Practice, 2016, Article ID: 9509705. https://doi.org/10.1155/2016/9509705

[26] Sen, S.S. and Rasul, E.S. (2006) Dermatophytosis in Assam. Indian Journal of Medical Microbiology, 24, 77-78. https://doi.org/10.4103/0255-0857.19907

[27] da Silva, B.C., Paula, C.R., Auler, M.E., Ruiz Lda, S., Dos Santos, J.I., Yoshioka, M.C., et al. (2014) Dermatophytosis and Immunovirological Status of HIV-Infected and AIDS Patients from Sao Paulo City, Brazil. Mycoses, 57, 371-376. https://doi.org/10.1111/myc.12169

[28] Costa, J.E., Neves, R.P., Delgado, M.M., Lima-Neto, R.G., Morais, V.M. and Coelho, M.R. (2015) Dermatophytosis in Patients with Human Immunodeficiency Virus Infection: Clinical Aspects and Etiologic Agents. Acta Tropica, 150, 111-115. https://doi.org/10.1016/j.actatropica.2015.07.012

[29] Havlickova, B., Czaika, V.A. and Friedrich, M. (2008) Epidemiological Trends in Skin Mycoses Worldwide. Mycoses, 51, 2-15. https://doi.org/10.1111/j.1439-0507.2008.01606.x

[30] Heidrich, D., Garcia, M.R., Stopiglia, C.D., Magagnin, C.M., Daboit, T.C., Vetoratto, G., et al. (2015) Dermatophytosis: A 16-Year Retrospective Study in a Metropolitan Area in Southern Brazil. The Journal of Infection in Developing Countries, 9 , 865-871.

[31] Courtellemont, L., Chevrier, S., Degeilh, B., Belaz, S., Gangneux, J.P. and Robert-Gangneux, F. (2017) Epidemiology of Trichophyton verrucosum Infection in Rennes University Hospital, France: A 12-Year Retrospective Study. Medical Mycology, 55, 720-724. https://doi.org/10.1093/mmy/myw142

[32] Nenoff, P., Kruger, C., Ginter-Hanselmayer, G. and Tietz, H.J. (2014) Mycology-An Update. Part 1: Dermatomycoses: Causative Agents, Epidemiology and Pathogenesis. JDDG: Journal der Deutschen Dermatologischen Gesellschaft, 12, 188 210. https://doi.org/10.1111/ddg.12245

[33] Costa-Orlandi, C.B., Magalhaes, G.M., Oliveira, M.B., Taylor, E.L., Marques, C.R. and de Resende-Stoianoff, M.A. (2012) Prevalence of Dermatomycosis in a Brazilian Tertiary Care Hospital. Mycopathologia, 174, 489-497. https://doi.org/10.1007/s11046-012-9576-1

[34] Persinoti, G.F., Martinez, D.A., Li, W., Dogen, A., Billmyre, R.B., Averette, A., et al. (2018) Whole-Genome Analysis Illustrates Global Clonal Population Structure of the Ubiquitous Dermatophyte Pathogen Trichophyton rubrum. Genetics, 208, 1657 1669. https://doi.org/10.1534/genetics.117.300573

[35] Drira, I., Neji, S., Hadrich, I., Trabelsi, H., Sellami, H., Cheikhrouhou, F., et al. (2014) Polymorphisms in the ITS rDNA Regions for Differentiating Strains of the Trichophyton mentagrophytes Complex in Sfax-Tunisia. Mycoses, 57, 453-459. https://doi.org/10.1111/myc.12181 


\section{Supplementary Material}

Table S1. List of the of the patients and isolates identified in the study containing the anatomical site, HIV status, CD4+ count, direct microscopy, phenotypic identification, ITS-RFLP and ITS sequencing.

\begin{tabular}{|c|c|c|c|c|c|c|c|c|c|}
\hline Patient & train ID & $\begin{array}{l}\text { Anatomical } \\
\text { site }\end{array}$ & Gender & HIV status & $\begin{array}{c}\text { CD4+ } \\
\text { T-cell count }\end{array}$ & $\begin{array}{c}\text { Direct } \\
\text { microscopy }\end{array}$ & $\begin{array}{l}\text { Phenotypic } \\
\text { identification }\end{array}$ & ITS-RFLP & Sequencing \\
\hline P23 & D01 & toenails & M & HIV-infected & 104 & positive & T. rubrum & T. rubrum & T. rubrum \\
\hline P27 & D04 & toenails & M & HIV-infected & 94 & positive & Trichophyton sp. & T. rubrum & not sequenced \\
\hline P201 & D09 & feet & F & HIV-infected & 542 & positive & T. rubrum & T. rubrum & not sequenced \\
\hline P85 & D10 & toenails & F & HIV-infected & 171 & negative & T. verrucosum & T. verrucosum & not sequenced \\
\hline P182 & D11 & toenails & M & HIV-infected & 1585 & positive & T. interdigitale & T. interdigitale & not sequenced \\
\hline P168 & D12 & feet & M & HIV-infected & 160 & positive & T. interdigitale & T. interdigitale & not sequenced \\
\hline P164 & D13 & arms & M & HIV-infected & 529 & negative & T. rubrum & T. rubrum & not sequenced \\
\hline P188 & D14 & inguinal & M & HIV-infected & 123 & positive & T. interdigitale & T. rubrum & not sequenced \\
\hline P194 & D16 & trunk & M & HIV-infected & 118 & positive & T. rubrum & T. rubrum & not sequenced \\
\hline P184 & D17 & toenails & F & HIV-infected & 1008 & positive & T. rubrum & T. rubrum & not sequenced \\
\hline P247 & $\mathrm{D} 21$ & toenails & M & HIV-infected & 427 & negative & T. rubrum & T. rubrum & not sequenced \\
\hline P202 & $\mathrm{D} 22$ & fingernails & M & HIV-infected & 876 & negative & Trichophyton sp. & not digested & Montagnulaceae sp. \\
\hline P109 & D23 & toenails & M & HIV-infected & 1105 & positive & T. rubrum & T. rubrum & T. rubrum \\
\hline P238 & D24 & toenails & M & HIV-infected & 74 & negative & T. interdigitale & T. rubrum & not sequenced \\
\hline P381 & D25 & feet & M & HIV-infected & 95 & positive & T. rubrum & T. rubrum & T. rubrum \\
\hline P235 & D26 & toenails & M & HIV-infected & 930 & positive & T. rubrum & T. rubrum & T. rubrum \\
\hline P405 & D34 & feet & $\mathrm{F}$ & HIV-infected & 730 & negative & T. rubrum & T. rubrum & not sequenced \\
\hline P15 & D41 & toenails & M & HIV-infected & 388 & positive & T. rubrum & T. rubrum & not sequenced \\
\hline P16 & D05 & hands & M & non-HIV & NA & positive & Trichophyton sp. & not digested & $\begin{array}{l}\text { Lecanicillium } \\
\text { aphanocladii }\end{array}$ \\
\hline P71 & D06 & arms & F & non-HIV & NA & negative & M. canis & M. canis & not sequenced \\
\hline P103 & D07 & trunk & $\mathrm{F}$ & non-HIV & NA & positive & Trichophyton sp. & not digested & Preussia sp. \\
\hline P123 & D15 & feet & $\mathrm{F}$ & non-HIV & NA & positive & T. rubrum & T. rubrum & not sequenced \\
\hline P185 & D18 & toenails & $\mathrm{F}$ & non-HIV & NA & positive & T. rubrum & T. rubrum & not sequenced \\
\hline P372 & D27 & fingernails & M & non-HIV & NA & positive & T. rubrum & T. rubrum & T. rubrum \\
\hline P390 & D28 & feet & F & non-HIV & NA & negative & T. rubrum & T. rubrum & not sequenced \\
\hline P384 & D29 & toenails & M & non-HIV & NA & positive & T. rubrum & T. rubrum & not sequenced \\
\hline P397 & D30 & feet & $\mathrm{F}$ & non-HIV & NA & positive & Trichophyton sp. & T. rubrum & not sequenced \\
\hline P382 & D32 & toenails & M & non-HIV & NA & positive & Trichophyton sp. & T. rubrum & not sequenced \\
\hline P403 & D33 & feet & F & non-HIV & NA & positive & T. interdigitale & T. interdigitale & T. interdigitale \\
\hline P222 & D35 & toenails & $\mathrm{F}$ & non-HIV & NA & negative & T. rubrum & T. rubrum & not sequenced \\
\hline P64 & D38 & feet & $\mathrm{F}$ & non-HIV & NA & positive & T. interdigitale & T. interdigitale & T. interdigitale \\
\hline P175 & $\mathrm{D} 40$ & toenails & M & non-HIV & NA & positive & T. rubrum & T. rubrum & not sequenced \\
\hline
\end{tabular}

NA: Not available. 